\title{
Acerca del valor teórico y metodológico de la categoría vygotskiana "Vivencia" para la Enseñanza
}

\author{
About the theoretical and methodological value of the Vygotskian \\ category "Experiencing" for Teaching
}

Maria Milagros Flebes Elejalde ${ }^{1}$

\begin{abstract}
RESUMEN
En el presente artículo se analiza la concepción optimista del hombre que ofrece el Enfoque Histórico Cultural y se valora la obra de su impulsor L. S. Vygotski. Se define la categoría "vivencia" y describen las transformaciones que atraviesa la vivencia como realidad que une al sujeto con su medio desde el nacimiento del niño hasta edades en que la enseñanza refuerza el desarrollo psíquico con la maduración de funciones que se relacionan entre sí. Se explican las funciones de la vivencia y sus características a la vez que se intenta demostrar la importancia o necesidad de que los docentes profundicen en su estudio por la relación con la enseñanza desarrolladora.
\end{abstract}

Palabras clave: Enfoque histórico-cultural. Vivencia. Enseñanza.

\begin{abstract}
This article analyzes the optimistic conception of man offered by the Cultural Historical Approach and the work of its promoter L. S. Vygotski is valued. The category "experience" is defined and they describe the transformations that the experience goes through as a reality that unites the subject with his environment from the birth of the child until the ages when teaching reinforces the psychic development with the maturation of functions that are related to each other. The functions of the experience and its characteristics are explained while trying to demonstrate the importance or need for teachers to deepen their study due to the relationship with developer teaching.
\end{abstract}

Keywords: Historical-cultural approach. Experience. Teaching.

\section{Introducción}

El Enfoque Histórico Cultural del desarrollo psíquico creado por L. S. Vygotski y sus seguidores, constituye el punto de partida excepcional para una concepción optimista del hombre y su educación, de ahí que resulta de gran importancia que este enfoque se aplique consecuentemente a la enseñanza. Esta concepción centra su interés en el desarrollo integral del hombre, donde un papel

\footnotetext{
1 Licenciada en Psicología en la Universidad de la Habana (1971), Doutora en las Ciencias Psicológicas en la Universidad Estatal de Moscú (1977), Master en Psicología Educativa (1998) y Master en Grupo y Psicodrama en la Universidad de la Habana (2009), Profesora Titular (1990) y atualmente Consultante de la Universidad de la Habana (2013). ORCID: https://orcid.org/00000002-2952-5500. E-mail: maria.febles@psico.uh.cu. mafebles49@gmail.com.
} 
fundamental juega la experiencia sociohistórico y cultural de la que es capaz el hombre de apropiarse. Su base filosófica es el materialismo dialéctico e histórico, el cual es asimilado por este autor de manera creadora y consecuente en el estudio del origen y desarrollo de las funciones psíquicas superiores, forma en que denominó a la psique humana, ya que para él lo psíquico es una función del ser corporal, histórico y social que es el hombre. Esta nueva concepción históricocultural de dichas funciones inauguró un nuevo paradigma en la ciencia psicológica. Su impulsor dio los primeros pasos, que, sin estar exentos de críticas y errores, vislumbró un camino seguro a seguir en la investigación. Vio con claridad la encrucijada metodológica en que se encontraba la Psicología, interpretó y enfrentó la crisis con una coherente propuesta que marcó la vía del crecimiento humano, como dirección a seguir.

Pensamos que este genial psicólogo inauguró una teoría del desarrollo de las funciones psíquicas superiores, que sirve de marco para la investigación no solo de la subjetividad humana, sino también para la de la Enseñanza. Creó fundamentos psicológicos no solo para esta ciencia, sino también para otras áreas de trabajo como la Pedagogía, Sociología, Medicina, Comunicación, Comercio y otras muchas.

En el caso que nos ocupa, la enseñanza, son varios los aportes científicos en los que descansa. Empezando por el sistema de categorías que se utilizan y que posee un valor teórico y metodológico incuestionable, ya que nos proporciona realidades, que muy convenientemente se aplican al proceso de enseñanzaaprendizaje. Si bien este enfoque no resulta una teoría acabada, si se considera la plataforma psicológica para la explicación del desarrollo humano. Conocemos que la enseñanza no ocurre paralelamente al desarrollo, ellos se entrecruzan de manera tal que la primera promueve cambios que estimulan el segundo. Todo ser humano cuenta con potencialidades de desarrollo que se hacen realidad cuando aparece lo nuevo que produce la enseñanza. Para una mayor y mejor comprensión de esto es necesario comenzar por la categoría vivencia, la cual por el lugar central que ocupa, exige su momento explicativo. 
Nos hacemos personalidad y no somos conscientes de nuestras mediaciones, de aquellos canales que nos enlazan no solo con la realidad física exterior, sino también con el desarrollo histórico y social de la sociedad en que nacemos. Los signos - en la teoría vygotskiana - son instrumentos, órganos, subjetivos de lo psíquico, mediante los cuales el hombre asimila los productos sociales. Entre las diferentes categorías que elabora L.S. Vygotski en su plataforma teórica se encuentra una que por la posición central que ocupa merece ser tratada especialmente. Se trata de la vivencia. Este término fue anteriormente utilizado por el Psicoanálisis, y comienza a ser tratado por él en su "Psicología del Arte" ocupando aquí también un lugar determinado. En su conferencia "El problema del Entorno" este autor destaca el papel, el significado y la influencia del entorno en el desarrollo del niño, afirmando que éste es visto en dependencia de la posición interna del niño y del tipo de relación que establece con ese entorno. Está claro que el niño no está igualmente desarrollado para interpretar el mundo al año, a los 18 meses, ni a los 3 años, ya que sus posiciones internas son diferentes y van a caracterizar el desarrollo de cada uno. Con este trabajo intentamos develar la necesidad de que los docentes conozcan la categoría "vivencia" de manera que puedan utilizarla en su trabajo docente y educativo con el objetivo de lograr una enseñanza desarrolladora.

\section{2 ¿Qué es, cómo surge y cambia la vivencia?}

Vygotski en su Psicología del Desarrollo destacó el papel importante del componente histórico-cultural en el surgimiento de las funciones psíquicas del hombre. Ya desde antes, filósofos marxistas advertían que la explicación de los hechos psicológicos había que buscarla fuera de ellos. Esta afirmación se comprende en tanto lo externo: la sociedad, su historia y la cultura, que, en un inicio, están fuera del sujeto, de su organismo, como fuentes proveedoras de los materiales a los que se enfrenta la subjetividad en formación. El hombre refleja, hace suyos los contenidos culturales en dependencia del nivel de desarrollo alcanzado. Y lo hace a través de un proceso mediado, selectivo, de intercambio y de interrelación dialéctica con lo 
externo. Este es el mecanismo de producción de la vivencia, la que refleja de modo particular lo interno y lo externo. Considerando a la realidad social como la primera fuente (léase fuente y no determinante) de desarrollo, la vivencia como hecho psicológico es un reflejo parcializado de la realidad histórico social externa a ella. Tiene una función selectiva, señalizadora, ya que hace posible que lo social significativo se transforme en individual, que lo social participe, signifique, y constituya la subjetividad humana.

De esta forma las vivencias son la unidad de lo interno y lo externo, ya que las mismas tienen como contenido lo externo, pero por su forma, reflejan las condiciones subjetivas internas. Han sido consideradas la unidad de la conciencia: "La verdadera unidad dinámica de la conciencia, unidad plena que constituye la base de la conciencia es la vivencia" (VYGOTSKI, 1994, p. 290). Son además la unidad de lo cognitivo y lo afectivo, ya que ponen en juego procesos emocionales y sensoperceptuales y de pensamiento, así como la esfera de los sentidos en dependencia de la edad psicológica de los sujetos vivenciales. Ellas se han considerado unidades de análisis de la Situación Social de Desarrollo, ya que a través de ellas se interioriza y refleja el contenido social, histórico y cultural del individuo de acuerdo con el nivel de desarrollo alcanzado. Tienen un papel muy significativo en las transformaciones del desarrollo, ya que a través del prisma de ellas se facilita o no, que se produzcan las contradicciones internas que llevan al cambio. Al respecto Vygotski escribió: “...la esencia de toda crisis reside en la reestructuración de la vivencia interior, reestructuración que radica en el cambio del momento esencial que determina la relación del niño con el medio..." (VYGOTSKI, 1994, p. 292), es decir, en el cambio de sus necesidades y motivos que son los motores de su comportamiento.

El estudio aislado de las vivencias puede describir, pero no explicar la estructura y dinámica de la personalidad. Para ello es necesario hablar de otra función de la vivencia que es su función reguladora, su papel en las leyes fundamentales del desarrollo, sus características y etapas. Para el cambio es necesario que la lógica de los procesos de desarrollo interno de la subjetividad haya alcanzado su nivel de desarrollo óptimo (período sensitivo), este nivel de 
desarrollo yace "subterráneo", inconsciente, latente como en actitud de aceptación de la incertidumbre ante lo nuevo (nuevos objetos o fenómenos), de manera que bajo la guía de un adulto, otro contemporáneo, o el nivel de desarrollo autorreflexivo alcanzado, y la vivencia de la contradicción, se pueda dar el salto cualitativo con la aparición de las nuevas formaciones de la edad.

La mayoría de estos saltos ocurren de una edad para otra del desarrollo, por ello en cada una es necesario el estudio de cómo el medio o entorno interviene en la dinámica particular a través de la vivencia. Ello deberá una correcta solución al papel de cómo cada uno participa en la dinámica de la edad. Es decir que en el inicio de cada edad deberá estudiarse la relación que se establece entre el sujeto y el entorno que lo rodea, sobre todo el social, lo que nos hace acudir a un concepto que nos da la clave en la teoría psicológica histórico-cultural: esta es la categoría Situación Social del Desarrollo. Esta constituye el contexto psicológico en que se dan todos los cambios dinámicos que se producen en el desarrollo durante el período de la edad. La Situación Social del Desarrollo podemos definirla como aquella relación peculiar, única, especial e irrepetible entre el sujeto y su entorno que va a determinar las líneas de desarrollo, la forma y trayectoria que permiten al mismo adquirir nuevas propiedades de la personalidad. Aclarando la Situación Social de Desarrollo, conoceremos realmente cual es la dinámica de desarrollo de la edad correspondiente, ya que la vivencia, su unidad, aporta un referente que sirve de filtro de los contenidos culturales, afín a su sistema y revela lo específico y único de la situación que influye sobre el niño o adulto en una etapa del desarrollo y contexto específico. Es espacio en que la comunicación y la actividad determinan el desarrollo de las potencialidades. No está de más aclarar el carácter francamente individual de la misma. La palabra social solo se refiere al origen de este proceso que inicia cada subjetividad. El sujeto abocado en una etapa de desarrollo se encuentra en una unidad de elementos personales y ambientales únicos, posee un sistema de vivencias y experimenta otras nuevas vivencias provenientes de sus relaciones con el mundo real, dinámico, del medio con el que interactúa y que se transforma constantemente como resultado del carácter cambiante de las relaciones 
psicológicas y sociales: económicas, laborales, organizacionales, familiares etc. Estos cambios son percibidos por el sujeto, pero no actúan linealmente, teniendo primero que penetrar en su sistema psicológico de representaciones, convirtiéndose, en contenidos psicológicos que en dependencia de ese nivel alcanzado entrará o no en contradicción con lo existente. Esto va creando las condiciones para la aparición de la crisis o etapas críticas.

Diferentes autores han analizado el papel de las condiciones externas en la producción de las crisis, observándose que la propia lógica interna del proceso de desarrollo (y el papel de las vivencias en ella) es la que provoca la necesidad de muchos períodos críticos, de viraje y no la presencia o ausencia de condiciones específicas en el entorno. Eso solo habla de un proceso interno que debe alcanzar cierto nivel de desarrollo para generar la contradicción. Las contradicciones se vivencian, es decir adquieren una expresión subjetiva ante los objetos y fenómenos del entorno que se relacionan de alguna forma con la satisfacción o insatisfacción de las necesidades que coexisten en la subjetividad y son las únicas capaces de revolucionar, ocasionar la reconstrucción o reestructuración en la subjetividad del sujeto, ocasionando movimientos en sus relaciones y dependencias interfuncionales de la personalidad existentes. Como ya se ha señalado la esencia de toda crisis reside en la reestructuración de la vivencia interior (sistema de vivencias), reestructuración que radica en el cambio del momento esencial que determina la relación con el medio, es decir, en el cambio del sentido, de las necesidades y motivos asociados a él y que son los que determinan la relación. El sujeto como sistema psicológico, como personalidad se resiste y ello crea las crisis: malestares, desequilibrios, revoluciones. No es fácil describir teóricamente este proceso, por ello solo se intenta una aproximación al afirmar la creación de contradicciones internas que marcan la aparición de lo nuevo.

El cambio entre una edad y otra puede entenderse así, dialécticamente decía Vygotski, a partir de las leyes de la negación de la negación y de la transformación de los cambios cuantitativos en cualitativos (o ley de los saltos) a los que contribuye la vivencia con la apertura a nuevos contenidos. La integración 
de algo nuevo niega lo viejo que era nuevo antes de la incorporación. De hecho, y en última instancia, la determinante principal del desarrollo es la solución de la acción mutua de las contradicciones internas inherentes al fenómeno; la ocurrencia de un automovimiento en todo lo existente. El desarrollo es aquella forma de activación que tiene un carácter interno, que constituye una unidad como vínculo interno entre estadios, es un movimiento dinámico, de lo simple a lo complejo, de lo inferior a lo superior, de lo viejo a lo nuevo, es cambio, siendo su motor el automovimiento que parte del impulso de solución de las contradicciones internas, que se formaron en la relación mutua con el medio circundante.

Si estamos de acuerdo en que, en el paso de una edad a otra, lo que se reorganiza es la estructura de la conciencia, la formación nueva principal de cada edad no es otra que el nuevo nivel de autoconciencia que se alcanza.

Es importante aclarar que L.S. Vygotski no incluyó la etapa de la juventud en el desarrollo infantil ya que la consideró el eslabón inicial en la cadena de edades maduras, más que el final de las edades infantiles. Es de nuestra opinión que las leyes del desarrollo son las mismas, aunque con nuevas complejidades, no existentes en épocas tempranas del desarrollo. Lo que cambian en las edades maduras, son los niveles cada vez más desarrollados de autoconciencia y de autorregulación que afectan todas las áreas de la personalidad. Ello reafirma la idea de que en el paso de una edad a otra lo que se modifica es la estructura general de la conciencia, la cual se distingue por un sistema determinado de relaciones y dependencias entre las funciones aisladas, entre las distintas formas de su actividad; por tanto, también cambian de lugar las líneas centrales y accesorias del desarrollo.

La función selectiva, señalizadora y reguladora de las vivencias juega un papel muy importante en el desarrollo psíquico, siendo imprescindible la reestructuración de las mismas para que se produzca el cambio. Entre las características que presentan las vivencias pudiéramos hablar de su contenido, carácter personal, intensidad y polaridad. La caracterización de las vivencias en los estudiantes constituye una problemática abierta a la enseñanza, que el estudio e investigación científica futura darán luz al respecto. 
El incremento y el cambio de las necesidades y motivos de la actividad de estudio es la parte menos consciente y voluntaria de la personalidad y a medida que el niño pasa de una edad a otra nacen en él nuevos impulsos. Es decir que la vivencia pasa de estados vivenciales, carentes de contenido objetivo en el recién nacido, a la vivencia del niño provocada por actividad conjunta con la madre en una situación concreta y en la que se diferencia, destaca y forma. Esta comunidad psíquica que antecede la conciencia se expresa en un complejo indiferenciado de vivencias emocionales provocadas por la madre en el primer año de vida, y que, junto a las otras personas, (padre, familia) va constituyendo, engrosando su mundo objetal y social hacia la edad temprana. A partir del año y medio de vida se producen cambios de actitud ante los objetos que se acercan y alejan apareciendo la atracción afectiva por ellos. Es la etapa de la aparición del gesto indicativo y con él las formas preverbales de comunicación. Y aunque la actitud ante el objeto y la persona todavía no están diferenciados en este bebé, no cabe duda de que es el otro (la madre, u otra persona), quien cambia el significado y el sentido de la situación misma, y por tanto de las vivencias. Es así que el mundo de sus propias vivencias internas es penetrado lenta y gradualmente por la sociedad, la historia y la cultura representados por su mamá.

La psique del bebé está incluida desde el primer momento de su vida en la existencia común con otras personas. A lo primero que reacciona el niño no es a sensaciones aisladas, sino a la gente de su entorno [...]. Incluso en edades posteriores perduran en el niño vestigios de esa insuficiente separación de su personalidad del todo social y del mundo circundante (VYGOTSKI, 1990, p.236).

En este sentido corrobora la idea de que la imitación de otras personas debe incluirse entre las peculiaridades específicamente humanas, ya que la compara con el animal que no puede aprender nada nuevo por medio de la imitación; sin embargo, el niño, por el contrario, adquiere gracias a ella nuevas formas de comportamiento inexistentes antes. Con los cambios de afectividad de las relaciones sociales del niño, hacia los 3 años aparecen las vivencias más profundas e intensas, sumergiéndose en una serie de conflictos internos y externos que le producen frecuentemente reacciones neuróticas. Se plantea que la 
crisis de los 3 años es una crisis de las relaciones sociales. No menos conflictiva llega a ser la crisis de los 7 años, que trata de un período de transición, donde el niño ya no es un preescolar pero tampoco escolar.

Ello acarrea como se ha visto dificultades en su educación, debido a la pérdida de su espontaneidad e ingenuidad. Empiezan a diferenciarse las facetas interior y exterior de la personalidad, apareciendo conductas extrañas, no del todo comprensibles, artificiales y hasta forzadas

[...] sobre todo en aquellos que han tenido una infancia difícil y cuyas vivencias de la crisis se manifiesta con mayor agudeza. El niño se amanera, se hace caprichoso, cambia de forma de andar. Se comporta de un modo artificioso, teatral, bufonesco, le gusta hacer el payaso (VYGOTSKI, 1990, p.286).

En contraposición a la acción ingenua y directa propia del niño preescolar, en la etapa siguiente se incorpora un elemento intelectual entre la vivencia y el acto directo del niño escolar, lo que hace que aparezca la vivencia atribuida de sentido. Esto quiere decir que a los siete años se forma en el niño una estructura de vivencias que le permite comprender lo que significa "estoy alegre o disgustado", ya que, al diferenciarse las facetas internas y externas, surge la orientación consciente de sus propias vivencias. Esto último no era posible en el niño de tres años, que solo descubre sus relaciones con otras personas. Ahora él descubre el propio hecho de sus vivencias, que se caracterizan por adquirir sentido ya que puede generalizarlas y reconocerlas internamente al punto de juzgarlas y concientizar intensas pugnas entre ellas.

Como hemos visto en esta edad escolar ya han aparecido y relacionado nuevas funciones que facilitan el proceso enseñanza-aprendizaje. Estas son: la percepción, la atención, la memoria y el pensamiento en las que la enseñanza se apoya para el aprendizaje de nuevos conocimientos y la formación del educando. El maestro, el profesor u orientador debe conocer las características de las edades psicológicas de sus educandos, así como los cambios en sus vivencias. La enseñanza exige de una buena preparación desde el punto de vista psicológico de los docentes de manera que puedan no solo formar y acumular conocimientos y 
habilidades, sino también desarrollar la personalidad de sus alumnos. La consideración de las vivencias en los educandos durante el proceso de enseñanza permite que el maestro regule el proceso en la dirección necesaria con su entorno, marcando momentos cruciales para su desarrollo futuro, determinando el desempeño del niño y la efectividad de su conducta. También a través de la vivencia se contribuye a formar rasgos del carácter y la personalidad. De su función señalizadora, el docente puede regular el curso de la enseñanza, hacerla más amena, práctica o teórica por su parte.

\section{Vivencia y enseñanza desarrolladora}

D. B. Elkonin, científico ruso muy cercano a Vygotski consideró necesario destacar con toda determinación, que las investigaciones teóricas generales de Vygotski sirvieron de base para el desarrollo de sus investigaciones especiales en el campo de la psicología propiamente infantil (evolutiva), no habiendo sido fácil su acercamiento, ya que lo hizo bajo las exigencias de la práctica como profesor, interesándose por las cuestiones de la psicología pedagógica incluso antes de que él se dedicara a investigar los problemas generales de la Psicología. Para él no hay dudas de que la elaboración de los problemas de enseñanza y desarrollo desempeñó un papel importante en la formación de las concepciones psicológicas generales de este autor (ELKONIN, 1994, p. 294).

Como se ha podido observar en el epígrafe anterior, la esencia de toda crisis reside en la reestructuración de la vivencia interior, que implica el cambio de la relación del niño con el medio, y con ello, el cambio de sus necesidades y motivos que son los motores de su comportamiento. La aparición de vivencias atribuidas de sentido está respaldada por el incremento y el cambio de necesidades y motivos. Estas son la parte menos consciente y voluntaria de la personalidad y en la medida que el niño pasa de una edad a otra nacen en él nuevos impulsos, nuevos motivos o, como diría nuestro autor, "los propulsores de su actividad experimentan un reajuste de valores" (VYGOTSKI, 1994, p.292).

L. S. Vygotski contrapone sus ideas a todas las teorías tradicionales sobre enseñanza-aprendizaje, insistiendo en que el proceso de desarrollo depende del 
carácter y contenido del propio proceso de la enseñanza y confirmando teórica y experimentalmente el papel rector de la enseñanza en el desarrollo mental de los niños. Una enseñanza dinámica que considere las peculiaridades del niño en su etapa constituiría una influencia real no solo para la formación de conocimientos, habilidades y hábitos, sino también para el desarrollo de su personalidad. Al respecto decía: "A mi juicio, toda vivencia está respaldada por una influencia real, dinámica, del medio con relación al niño" (VYGOTSKI, 1996, p. 292). En este sentido la enseñanza creará ambientes de comunicación que formen y desarrollen vivencias positivas en el aula, trabajará con las motivaciones de sus educandos y con el material a impartir.

El hombre se diferencia del animal por su capacidad de aprendizaje, pero éste debe realizarse en condiciones favorables para el aprendizaje. El camino de la formación y perfeccionamiento del hombre quedó claro desde hace más de un siglo, que no dependía en lo fundamental, de los mecanismos de la herencia, sino en gran medida de la apropiación de la experiencia externa como resultado de la interacción con el medio. Medio que es social e histórico cultural y como tal fuente de su desarrollo. El hombre aprende cualitativamente mejor y cuantitativamente más que el animal y lo hace no por las mismas vías comunes con él (herencia biológica, formación de reflejos condicionados), sino además por la apropiación de la experiencia histórico social y cultural acumulada como resultado del intercambio y reflejo personalizado de la realidad a lo largo de toda su vida. Gracias a la enseñanza se engendra la compleja subjetividad humana, aquella especialmente diferente, genuinamente social, genéticamente cultural e histórica y que en determinada medida se elabora con el activismo del propio sujeto que aprende. Este papel de la enseñanza se expresa cuando en un inicio el niño o joven aprende nuevas acciones con la ayuda y dirección del maestro, pero más tarde las realiza de forma independiente.

Aparece aquí otra categoría vygotskiana muy relacionada con la vivencia y es la de Zona de Desarrollo Próximo (ZDP). Esta es la zona subjetiva que se encuentra entre lo que el alumno puede hacer con la ayuda 
del maestro - mediante la imitación, corrección, demostración, señalamiento y lo que posee éste en su actividad autónoma. Los niveles de ayuda que aporta el maestro en la clase y la participación activa del alumno reducen la distancia entre estos límites, convirtiendo la habilidad potencial en real. Sin embargo, para que la enseñanza sea desarrolladora debe tener en cuenta las vivencias que expresan sus educandos. Estas se reflejan en los ambientes educativos, en la edad psicológica de sus estudiantes (cualidades de la percepción, pensamiento, atención y memoria) y el maestro debe adelantarse a los procesos, dirigiendo de la mejor forma los contenidos a aprender.

También muy relacionada está la categoría Período Sensitivo, que es aquel periodo evolutivo en que determinadas influencias educativas actúan más y mejor sobre el desarrollo psíquico que en otros. Muy conocido es el periodo en que con más fuerza se desarrolla el lenguaje y que es entre el año y medio y los 3 años. Si se aprovechan adecuadamente estas condiciones podemos hablar de una Enseñanza Desarrolladora a diferencia de otras en que se agota la ZDP, atiborrando de conocimientos desordenados e informaciones al niño. Esto puede convertirse en una simple acumulación que no se interioriza. ¿Y qué interioriza el alumno? A partir de los 7 años en que la vivencia es atribuida de sentido podemos afirmar que el alumno aprende aquello que se relaciona con su sistema de vivencias, intereses y motivaciones, por lo que sus vivencias deben ser interpretadas por el maestro. La enseñanza desarrolladora debe presentar al niño problemas que se hagan más complejos paulatinamente, y le exija un conocimiento independiente, para ello hay que enseñarle métodos y procedimientos, que investiguen y descubran los nuevos objetos y saquen conclusión con sus resultados. En todo esto es importante la introducción de acciones de orientación, que en la mayoría de los casos se resumen en el planteamiento de objetivos interesantes a alcanzar.

La enseñanza y educación son procesos que seleccionan, organizan planifican la experiencia histórico social y cultural acumulada de generaciones anteriores y que aprovechan las posibilidades de desarrollo alcanzado hasta el momento por el individuo (educando) permitiendo un fructífero y mejor 
aprendizaje. En esto no se excluye el papel de los mecanismos biológicos o hereditarios que se manifiestan y que constituyen premisas o condiciones anátomo-fisiológicas imprescindibles para su realización. Es decir que el aprendizaje constituye un proceso multifactorialmente determinado, siendo un aspecto destacado en nuestra bibliografía sobre el tema, el del papel activo del sujeto que aprende, éste, se constituye así, en un factor determinante más del proceso que analizamos. En él consideramos el cuerpo humano como mediador, al ser portador de vivencias y emociones que experimenta y que muchas veces pueden ser contradictorias entre sí y con lo que se siente y piensa. El cuerpo en su lenguaje traduce los sentidos psicológicos inconscientes que se esconden tras las vivencias (ZEIGARNIK; BRATUS, 1980). Por eso es importante concientizar su participación, sobre todo en el aspecto comunicativo del proceso.

El cuerpo media al constituir una unidad de análisis e intervención, que sirve para investigar sus propiedades y la detección de las potencialidades del sujeto, en términos de fomentar su activismo hasta que logre su pleno desarrollo e independencia. En trabajos anteriores apuntamos al tema de la mediación de madres, padres, maestros y otras figuras de relevancia en el desarrollo psíquico, queriendo significar que no solo las características psicológicas de las mismas, que intervienen en la actividad y comunicación, sino también las físicas, fisiológicas y otras del cuerpo humano también intervienen en la comunicación o percepción mutua que se establece en el proceso de enseñanza. Es decir que se establece una relación además de interpsicológica, inter corporal, comunicacional donde intervienen diferentes dimensiones que potencian favorablemente la enseñanza en su relación de ayuda o colaboración, potenciando elementos dinámicos internos que al poner en contradicción promoverán el cambio en la dirección deseada.

En el enfoque histórico-cultural se entiende por enseñanza aquel proceso en que el individuo humano organiza, promueve y guía determinada experiencia histórico-social para el estudiante y éste a la vez se apropia de la misma con su carácter activo. Esto último implica la existencia de un sujeto activo, que le da sentido a esta experiencia, transformándola en su subjetividad; es decir, que la 
enseñanza es un proceso que, partiendo de la experiencia, del medio externo como fuente proveedora, se dirige al enriquecimiento del individuo atendiendo a sus necesidades. Proceso que tiene como principios la actividad y la comunicación propias y de los otros. Proceso que es portador de toda la riqueza individual, cultural, histórica y social. Lo aprendido llega a ser objetivado en los productos de la actividad del sujeto y subjetivado a través del prisma de su sistema de vivencias subyacente en la conciencia individual.

En este proceso de la enseñanza se logra definir una amplia gama de procederes (zona de trabajo) para el maestro y otra zona de desarrollo próximo para el estudiante (ZDP) zona de potencialidades y posibilidades. El maestro activa las potencialidades del alumno en la relación interpersonal con su actividad y comunicación educativa logrando que potencialidades del alumno afloren a lo interno, intrapsíquicamente. La enseñanza no ocurre paralelamente al desarrollo, ellos se entrecruzan en el pasaje del nivel potencial al nivel real, siendo lo fundamental lo nuevo que el estudiante aprende. Los contenidos a aprender por el hombre están determinados por un mundo más vasto que va mucho más allá de lo que desde fuera actúa en el presente y ha actuado en el pasado, ya que a ellos se integran los contenidos que se han elaborado por la propia subjetividad individual a partir de ellos. El proceso se rige por las motivaciones, por las condiciones, la tarea del maestro es la de formar una conciencia desarrolladora de la personalidad de cada alumno, con vistas a su autodeterminación.

El enfoque histórico-cultural se nos presenta como el de mayores perspectivas de explicación y aplicación del desarrollo a través de la enseñanza. Desde este enfoque un aspecto importante que ha sido contemplado en el aprendizaje lo constituye el de sus bases fisiológicas. Al hablar de los mecanismos sobre los cuales descansa el comportamiento aprendido destaca la actividad condicionada y junto a ella, la noción de aferentación de retorno, la cual surge como resultado de un conjunto de condiciones fisiológicas y que permiten explicar una de las principales manifestaciones del aprendizaje, que es el perfeccionamiento de la estructura 
de los actos motores completos durante el curso de su realización. Perfeccionamiento que también tiene lugar en el plano de las acciones mentales y no se reduce a la formación de hábitos ya que estos no son la causa del comportamiento (LEONTIEV, 1977), es decir, que el aprendizaje se realiza por mecanismos funcionales especiales, que expresan las relaciones de los componentes del comportamiento. Especialmente en el hombre entran a jugar otros mecanismos de carácter psicológico que poseen una mayor relevancia en su comportamiento. Los actos humanos son actos intencionales que exigen una nueva forma de reflejo de la realidad: la conciencia. Sólo ella puede regular el acto y enfrentar manifestaciones objetivas. Las imágenes, representaciones e ideas existen para el hombre como realidades de las que se puede percatar y en relación con las que puede actuar y regular su comportamiento, como substrato funciona el lenguaje que representa un conjunto de abstracciones de los objetos mismos, bajo una forma material capaz de actuar sobre el propio sujeto portador. En resumen, en la enseñanza participan mecanismos fisiológicos conocidos y un sistema de realidades psicológicas coexistentes a conocer por los docentes que potencian y crean nuevos enlaces entre funciones en la personalidad de sus educandos.

En el hombre la asimilación de la experiencia histórico-social se adquiere junto a la experiencia individual, desde la más temprana edad la vida del niño. Con la enseñanza la experiencia de generaciones anteriores se organiza, planifica y realiza guiada por el adulto. Principios explicativos son los procesos de actividad y comunicación entre los hombres y con objetos del mundo, éstos permiten un análisis más profundo de la enseñanza y su papel en el desarrollo psíquico humano.

\section{Conclusiones}

- Hasta aquí solo hemos reflexionado en torno a la importante categoría Vivencia del Enfoque Histórico Cultural y su lugar en el proceso de la enseñanza y desarrollo, lo que deberá seguirse profundizando en la práctica e investigación pedagógica. 
- En el trabajo hemos demostrado cómo lenta y gradualmente la vivencia sufre cambios en dependencia de la edad psicológica, los métodos y procedimientos, aplicación de otras categorías también importantes. La atribución de sentido a las vivencias durante el proceso le otorga elementos motivacionales imprescindibles para el proceso de enseñanza-aprendizaje, así como la aplicación de categorías como la ZDP y Período sensitivo, que hay que tener en cuenta en el proceso de la enseñanza desarrolladora.

- La función señalizadora y reguladora de las vivencias juegan un papel muy importante en la enseñanza desarrolladora y su interpretación práctica por los maestros y profesores deben perfeccionar y profundizar su labor, investigando en el mismo proceso activo y dinámico de la enseñanza.

- La formación y desarrollo de vivencias comienza con la relación madreniño desde los primeros días de vida, continúa en el seno familiar, y en los siguientes grupos de los que forma parte este sujeto que aprende y se desarrolla.

- La enseñanza representa uno de los caminos más seguros del desarrollo si se concientiza la necesidad de una enseñanza desarrolladora.

-Solo desde un enfoque dialéctico que contempla la integración de todos los factores que intervienen en el proceso de enseñanza-aprendizaje se desarrolla la personalidad del estudiante.

\section{Referências}

ELKONIN D. B. Epílogo de Obras Escogidas de L. S. Vygotski. T4. Editorial Visor, Madrid, 1994.

ESPINOSA, B. En VYGOTSKI L. Psicología del arte. Editorial Pueblo y Educación. 1987.

LEONTIEV, A.N. La imagen del mundo. En: Obras psicológicas escogidas. T2 (en ruso) Ed. Pedagógica, Moscú, 1983.

VYGOTSKI, L. Obras Escogidas. Tomo 1. Editorial Visor, Madrid, 1990.

ZEIGARNIK V. y BRATUS B. Las formaciones de sentido. En: El desarrollo anormal de la Personalidad. Selección de lecturas de desarrollo moral. Compiladoras González O. y Martínez G., 1987. 


\section{Bibliografia}

BRATUS, B.S.; M. FEBLES El problema de la especificidad de las necesidades. En: Problemas teóricos y metodológicos de la personalidad. Ed. Pueblo y Educación, 1982.

BRATUS, B.S. La teoría psicológica general de la actividad y el problema de las unidades de análisis. En: A.N. Leontiev y la psicología contemporánea. Editorial Universidad de Moscú, Moscú, 1983.

GONZALEZ, R.F. Psicología de la Personalidad. Ed. Pueblo y Educación, La Habana, 1985.

LEONTIEV, A.N. Lo biológico y lo social en la psiquis del hombre. En: Problemas del desarrollo del psiquismo. Ed. Pueblo y Educación. Ciudad de La Habana, 1975.

LEONTIEV, A.N. Reflejos condicionados aprendizaje y conciencia. En: Seis conferencias sobre conceptos básicos de Psicología general. EMPES, 1977.

PETROVSKY, A.V. y V.A. PETROVSKY. La personalidad y su carácter activo a la luz de las ideas de A.N. Leontiev. En: La Psicología contemporánea. Ed. Universidad de Moscú, Moscú, 1983.

VYGOTSKI L. S Historia del desarrollo de las Funciones Psíquicas Superiores. La Habana: Científico Técnica, 1987.

VYGOTSKI L. S. El problema del entorno. Material traducido y fotocopiado, tomado del Vygotsky Readers, 1994.

VYGOTSKI L. S. Psicología del arte, La Habana, Pueblo y Educación, 1987.

VYGOTSKI L. S. Obras Completas, t.5, La Habana, Pueblo y Educación, 1989.

VYGOTSKI L. S. Obras Escogidas, t. 1. Madrid, Visor, 1990.

VYGOTSKI L. S. Obras Escogidas, t. 4. Madrid, Visor, 1994.

VYGOTSKI L. S. Obras Escogidas, t. 6. Madrid, Visor, 1996. 